\title{
PROPER HOLOMORPHIC MAPPINGS FROM THE TWO-BALL TO THE THREE-BALL
}

\author{
J. A. CIMA AND T. J. SUFFRIDGE
}

\begin{abstract}
We prove that a proper mapping of the two ball in $\mathbf{C}^{n}$ into the three ball, which is $C^{2}$ on the closed two ball is equivalent to one of four normalized polynomial mappings. This improves the known result of Faran. The proof is basic using Taylor expansions.
\end{abstract}

\section{INTRODUCTION}

Let $\Omega$ and $D$ be regions in $\mathbf{C}^{n}$ and $\mathbf{C}^{k}$ respectively. A holomorphic mapping $f: \Omega \rightarrow D$ is said to be a proper holomorphic mapping provided $f^{-1}(K)$ is a compact subset of $\Omega$ whenever $K$ is a compact subset of $D$. We use the following notation. Let $\langle z, w\rangle=z_{1} \bar{w}_{1}+z_{2} \bar{w}_{2}+\cdots+z_{n} \bar{w}_{n}$ be the inner product of $z, w \in \mathbf{C}^{n}$, denote the corresponding norms by $\|z\|^{2}=\langle z, z\rangle$ and let $B_{n}=\left\{z \in \mathbf{C}^{n}:\|z\|<1\right\}$.

If $f: \Delta \rightarrow \Delta$ is a proper holomorphic mapping where $\Delta=B_{1}=$ the unit disk in the complex plane, then it is easy to check that $f$ is a finite Blaschke product. Alexander [1] showed that if $f: B_{n} \rightarrow B_{n}$ is a proper holomorphic mapping with $n \geq 2$ then $f$ is a holomorphic automorphism of $B_{n}$ onto $B_{n}$.

Let $\phi$ and $\psi$ be holomorphic automorphisms of $B_{n}$ and $B_{k}$ respectively. Then $f: B_{n} \rightarrow B_{k}$ is a proper holomorphic mapping if and only if $g=\psi \circ f \circ$ $\phi: B_{n} \rightarrow B_{k}$ is a proper holomorphic mapping. Under these conditions, we say that $f$ is equivalent to $g$ and we write $f \sim g$. Thus, since the holomorphic automorphisms of $B_{n}$ are known, there is no loss in generality in assuming $f(0)=0$. Further, if $\min \left\{\operatorname{rank} D f(z): z \in B_{n}\right\}$, occurs at a point $z_{0}=B_{n}$, we may assume without loss of generality (in our characterization of proper maps) that $z_{0}=0$. This is true because $\phi$ and $\psi$ above can be chosen so that $\phi(0)=z_{0}$ and $\psi \circ f\left(z_{0}\right)=0$. Then $g \sim f, g(0)=0$ and

$$
\operatorname{rank} D g(0)=\operatorname{rank} D \psi\left(f\left(z_{0}\right)\right) \circ D f\left(z_{0}\right) \circ D \phi(0)=\operatorname{rank} D f\left(z_{0}\right)
$$

because $D \psi\left(f\left(z_{0}\right)\right)$ and $D \phi(0)$ are invertible.

We assume from this point on, that $f: B_{n} \rightarrow B_{k}$ is proper and holomorphic, $2 \leq n<k$ and that $f(0)=0$.

Received by the editors September 1, 1987.

1980 Mathematics Subject Classification (1985 Revision). Primary 32H99.

The first author would like to dedicate this work to his teachers, Professor H. Krall, Josephine Mitchell and I. Stright. 
Webster [5], proved that if $F: \bar{B}_{n} \rightarrow \bar{B}_{n+1}(\bar{A}$ is the closure of $A)$, yields a $C^{3}$ immersion of $\partial B_{n}$ into $\partial B_{n+1}, n \geq 3$, and $F$ is holomorphic on $B_{n}$ then $F\left(\bar{B}_{n}\right)$ is contained in an $n$-dimensional affine space. Thus, under our assumptions on $f$, if $3 \leq n, k=n+1$ and $f$ extends to a $C^{3}$ mapping of $\bar{B}_{n}$ into $\bar{B}_{n+1}$ then Webster's result says that $f$ is equivalent to the mapping $\left(z_{1}, z_{2}, \ldots, z_{n}\right) \rightarrow\left(z_{1}, z_{2}, \ldots, z_{n}, 0\right)$. Faran [3], then showed that if $n=2$, $k=3$ and $f$ extends to a $C^{3}$ mapping of $\bar{B}_{2}$ into $\bar{B}_{3}$ then $f$ is equivalent to one of the following four mappings.

$$
\begin{aligned}
\text { (i) } \quad(z, w) & \rightarrow(z, w, 0), \\
\text { (ii) } \quad(z, w) & \rightarrow\left(z, z w, w^{2}\right), \\
\text { (iii) } \quad(z, w) & \rightarrow\left(z^{2}, \sqrt{2} z w, w^{2}\right), \text { or } \\
\text { (iv) } \quad(z, w) & \rightarrow\left(z^{3}, \sqrt{3} z w, w^{3}\right) .
\end{aligned}
$$

In [2], the present authors showed that Webster's and Faran's results were true under the weaker assumption that $f$ extends to a $C^{2}$ mapping of $\bar{B}_{n} \rightarrow \bar{B}_{n+1}$ ( $n \geq 3$ for Webster's result and $n=2$ for Faran's result). We further showed that if $f$ extends to a $C^{2}$ mapping on $\overline{N \cap B_{n}}$ where $N$ is an open subset of $C^{n}$ with $N \cap \partial B_{n} \neq \phi, k \leq n(n+1) / 2$ and for each $u \in N \cap \partial B_{n}$, the set of vectors

$$
\{f(u)\} \cup\left\{D f(u)\left(v_{j}\right): 2 \leq j \leq n\right\} \cup\left\{D^{2} f(u)\left(v_{l}, v_{p}\right):(l, p) \in K\right\}
$$

is linearly independent then $f$ is a rational function. Here $u=\left(u_{1}, u_{2}, \ldots\right.$, $\left.u_{n}\right), u_{1} \neq 0$ and $v_{2}=\left(\bar{u}_{2},-\bar{u}_{1}, 0, \ldots, 0\right), v_{3}=\left(\bar{u}_{3}, 0,-\bar{u}_{1}, 0, \ldots, 0\right), \ldots$, $v_{n}=\left(\bar{u}_{n}, 0, \ldots, 0,-\bar{u}_{1}\right), 2 \leq j \leq n$, and $K$ is an appropriate set of $k-n$ pairs of integers $(l, p)$. Thus (2) is a collection of $k$ linearly independent vectors in $\mathbf{C}^{k}$. We further showed that $f=P / S$ where $P$ is a (vector-valued) polynomial of degree $\leq 2 k-n-1$ and $S$ is a polynomial of degree $\leq \operatorname{deg} P-1$ (assuming $f(0)=0=P(0)$ ). We conjectured that Webster's result could be improved to state that if $2<n<k \leq 2 n-2$ and $f$ extends to a $C^{2}$ mapping of $\bar{B}_{n}$ into $\bar{B}_{k}$ then $f$ is equivalent to the trivial mapping

$$
\left(z_{1}, z_{2}, \ldots, z_{n}\right) \rightarrow\left(z_{1}, z_{2}, \ldots, z_{n}, 0,0, \ldots, 0\right) .
$$

This would be the best possible result (as far as the size of $k$ is concerned) since the mapping $f: B_{n} \rightarrow B_{2 n-1}$ given by

$$
f\left(z_{1}, z_{2}, \ldots, z_{n}\right)=\left(z_{1}, z_{2}, \ldots, z_{n-1}, z_{1} z_{n}, z_{2} z_{n}, \ldots, z_{n}^{2}\right)
$$

is a proper holomorphic mapping. Faran [4] has shown that this conjecture is true under the stronger assumption that $f$ extends to a holomorphic mapping from an open set $N \subset \mathbf{C}^{n}, \bar{B}_{n} \subset N$, into $\mathbf{C}^{k}$.

Faran's nice results concerning proper holomorphic maps from $B_{2}$ into $B_{3}$ [3] were obtained by using moving frames and doing some long and tedious computations in projective space. In this paper, we obtain Faran's results by elementary means starting from our results in [2] which were also obtained by 
elementary means. We believe the method used here (i.e. minimizing $\|D g(0)\|$ over all $g$ equivalent to $f$ with $g(0)=0$ ) may be useful for characterizing the proper maps $f: B_{n} \rightarrow B_{k}$ for other $n$ and $k$. As described above, our main result is as follows.

Main Theorem. If $f: B_{2} \rightarrow B_{3}$ is a proper holomorphic mapping that extends to a $C^{2}$ mapping of $\bar{B}_{2}$ into $\bar{B}_{3}$ then $f$ is equivalent to one of the four mappings given in (1).

\section{PreliminaRY ResUlts}

In [2, Theorem 3], we showed that if $n=2, k=3$ and $f$ extends to be a $C^{2}$ map of $\bar{B}_{2}$ into $\bar{B}_{3}$ and $f$ does not satisfy the linear independence conditions of our main theorem then $f$ is equivalent to (l)(i). Therefore, we need only consider

$$
f=\frac{1}{S} P, \quad P(0)=0, \quad S(0)=1, \quad \operatorname{deg} S \leq \operatorname{deg} P-1 \leq 2 .
$$

The fact that $\operatorname{deg} S \leq \operatorname{deg} P-1$ follows from the fact that

$$
\left\langle P(\lambda u), P\left(\frac{1}{\bar{\lambda}} u\right)\right\rangle=S(\lambda u) \overline{S\left(\frac{1}{\bar{\lambda}} u\right)}
$$

when $\|u\|=1, \lambda$ a complex number, upon equating coefficients of the highest power of $\lambda$.

We will always assume, unless otherwise stated, that $\lambda$ is complex, $u \in \mathbf{C}^{2}$, $\|u\|=1$ and $v=\left(\bar{u}_{2},-\bar{u}_{1}\right)$ so that $\|v\|=1$ and $\langle u, v\rangle=0$. For any holomorphic function $h(z, w),(z, w) \in B_{2}$, we define $h^{*}(z, w)=\overline{h(\bar{w},-\bar{z})}$ so $h^{*}$ is also holomorphic and we note that

$$
h^{* *}(z, w)=h(-z,-w) .
$$

Since $f$ is rational, it can be expanded about almost every boundary point. From [2, Lemma 1], it follows that $\langle f(u), f(u+\lambda v)\rangle \equiv 1$. Thus,

$$
\langle P(u), P(u+\lambda v)\rangle=S(u) \overline{S(u+\lambda v)} .
$$

Hence we see that if $H$ is a vector-valued homogeneous polynomial consisting of the highest degree terms of $P$, then

$$
\langle P(u), H(v)\rangle=0
$$

because this is the coefficient of the highest degree terms in $\bar{\lambda}$. Since $\langle P(z, w)$, $\left.\overline{H^{*}(z, w)}\right\rangle$ is holomorphic for $(z, w) \in B_{2}$ (in fact for all $(z, w)$ since $P$ and $H^{*}$ are polynomials) and equals zero when $\|(z, w)\|=1$, we conclude

$$
0=\left\langle P(z, w), \overline{H^{*}(z, w)}\right\rangle=\sum_{j=1}^{3} P_{j}(z, w) H_{j}^{*}(z, w)
$$

for all $(z, w)$. 
Now let $L$ consist of the linear terms of $P$. By proper choice of coordinates, we may assume $L(z, w)=(a z+c w, b w, 0)$. In fact, since there exist perpendicular, nonzero vectors $(z, w),(\bar{w},-\bar{z})$ such that $\langle L(z, w), L(\bar{w},-\bar{z})\rangle=0$, we may find unitary maps $U$ and $V$ on $\mathbf{C}^{2}$ and $\mathbf{C}^{3}$ respectively such that $V \circ f \circ U$ has linear part $(a z, b w, 0)$ with $1 \geq a \geq 0$ and $1 \geq b \geq 0$. We will assume $f=P / S$ has this form when it is convenient to do so.

The difficulty in proving $f$ is equivalent to one of the mappings (1) is that it is not always easy to recognize $f$ as being $\psi \circ g \circ \phi$ where $g$ is one of the mappings (1) and $\phi$ and $\psi$ are biholomorphic automorphisms of $B_{2}$ and $B_{3}$ respectively. Therefore, we first consider briefly the effect of forming $\psi \circ f \circ \phi$. Let $Z \in B_{2}$ and $W \in B_{3}, r>1, u$ fixed, $u \in \partial B_{2}$ and $R=\|f(r u)\|$. We define $\phi$ and $\psi$ so that $\phi(0)=r u$ and $\psi(f(r u))=0$.

$$
\begin{aligned}
& \phi(Z)=\frac{1}{1+r\langle Z, u\rangle}(\langle Z+r u, u\rangle u\left.+\sqrt{1-r^{2}}(Z-\langle Z, u\rangle u)\right), \\
& \psi(W)=\frac{1}{1-\langle W, f(r u)\rangle}\left(\left\langle W-f(r u), \frac{1}{R} f(r u)\right\rangle \frac{f(r u)}{R}\right. \\
&\left.\quad+\sqrt{1-R^{2}}\left(W-\left\langle W, \frac{1}{R} f(r u)\right\rangle \frac{f(r u)}{R}\right)\right) .
\end{aligned}
$$

Now write $Z=\alpha u+\beta v$. Then

$$
\begin{aligned}
\phi(\alpha u+\beta v) & =\frac{\alpha+r}{1+\alpha r} u+\frac{\sqrt{1-r^{2}}}{1+\alpha r} \beta v \\
& =r u+\left(1-r^{2}\right) \alpha u+\sqrt{1-r^{2}} \beta v+o_{1}(\alpha, \beta) \\
f \circ \phi(\alpha u+\beta v)= & f(r u)+\left(1-r^{2}\right) \alpha D f(r u)(u) \\
& +\sqrt{1-r^{2}} \beta D f(r u)(v)+o_{2}(\alpha, \beta)
\end{aligned}
$$

and

$$
\begin{aligned}
\psi \circ f \circ \phi(\alpha u+\beta v)= & \frac{1-r^{2}}{1-R^{2}} \alpha\langle D f(r u)(u), U\rangle U \\
& +\frac{1-r^{2}}{\sqrt{1-R^{2}}} \alpha(D f(r u)(u)-\langle D f(r u)(u), U\rangle U \\
& +\frac{\sqrt{1-r^{2}}}{1-R^{2}} \beta\langle D f(r u)(v), U\rangle U \\
& \left.+\frac{\sqrt{1-r^{2}}}{\sqrt{1-R^{2}}} \beta(D f(r u)(v))-\langle D f(r u)(v), U\rangle U\right) \\
& +o_{3}(\alpha, \beta)
\end{aligned}
$$

where $U=f(r u) / R$ and we have used the fact that

$$
\frac{1}{1-\langle f(r u)+O(\alpha, \beta), f(r u)\rangle}=\frac{1}{1-R^{2}+O(\alpha, \beta)}=\frac{1}{1-R^{2}}+O(\alpha, \beta) .
$$


Here, $\left\|o_{j}(\alpha, \beta)\right\| /\|(\alpha, \beta)\| \rightarrow 0$ and $O(\alpha, \beta) /\|(\alpha, \beta)\|$ is bounded as $(\alpha, \beta) \rightarrow$ $(0,0)$. Set $g_{r u}=\psi \circ f \circ \phi$. Assume a sequence of $r_{n} \rightarrow 1$ and $u_{n},\left\|u_{n}\right\|=1$ are chosen so that $r_{n} u_{n} \rightarrow u$ and $\lim _{n \rightarrow \infty} g_{r_{n}} u_{n} \rightarrow g$ uniformly on compact subsets of $B_{2}$. Then clearly $g$ is holomorphic, $g(0)=0$ and $\|g(Z)\|<1$ for all $Z \in B_{2}$. Further,

$$
\frac{1-r_{n}^{2}}{1-R_{n}^{2}} \rightarrow \frac{1}{\langle D f(u)(u), f(u)\rangle} \quad\left(\text { when } R_{n}=\left\|f\left(r_{n} u_{n}\right)\right\|\right)
$$

so the first term on the right in (10) has limit $\alpha f(u)$. Since the first and third terms on the right lie in the same one dimensional space perpendicular to the second and fourth terms, the third term must have limit zero (if the limit is $l$, then

$$
\|g(\alpha u+\beta v)\| \geq\|\alpha f(u)+\beta l\|=|\alpha+c \beta|>\sqrt{|\alpha|^{2}+|\beta|^{2}}
$$

for some choice of $\alpha$ and $\beta$ unless $c=0)$. It follows that

$$
\frac{1}{\sqrt{1-R_{n}^{2}}}\left\langle D f\left(r_{n} u_{n}\right)\left(v_{n}\right), f\left(r_{n} u_{n}\right)\right\rangle \rightarrow 0 \quad \text { as } n \rightarrow \infty .
$$

Since $\|D f(u)(v)\|^{2}=\langle D f(u)(u), f(u)\rangle,[2$, Lemma 1(b)], the fourth term has limit $\beta D f(r u)(v) /\|D f(r u)(v)\|$ while the second term clearly has limit zero. Thus, it is easy to see that $g$ is a linear isometry. In fact, the same argument shows that even without the assumption $\lim _{n \rightarrow \infty} g_{r_{n} u_{n}}=g$ uniformly on compact subsets of $B_{2}$, we still have $D g_{r_{n} u_{n}}(0) \rightarrow L$ where $L$ is a linear isometry. Since holomorphic automorphisms are always of the form (9) up to composition with an appropriate unitary map, we have proved the following lemma.

Lemma 1. Let $f$ be a given proper holomorphic mapping of $B_{n}$ into $B_{k}$ that extends to a $C^{1}$ mapping of $\bar{B}_{n}$ into $\bar{B}_{K}$ with $f(0)=0$. Let $g_{\psi, \phi}=\psi \circ f \circ \phi$ where $\phi$ and $\psi$ are holomorphic automorphisms of $B_{n}$ and $B_{k}$ respectively with $g_{\psi, \phi}(0)=0$. If $\phi$ varies so that $\phi(0) \rightarrow \partial B_{n}$ then $D g_{\psi, \phi}(0) \rightarrow L$ where $L$ is a linear isometry. Further, there exists $\phi_{0}, \psi_{0}$ so that $\left\|D g_{\psi_{0}, \phi_{0}}(0)\right\| \leq\left\|D g_{\psi, \phi}(0)\right\|$ for all $\phi$ and $\psi$ as above.

The result is clearly true for general $n$ and $k$ because the dimension $k$ of the range space does not enter into the proof while the proof for the domain $B_{2}$ can be applied, viewing the vector $v$ in the proof as being any unit vector perpendicular to $u$.

It is clear that we may choose $f$ so that

$$
\|D f(0)\| \leq\left\|D g_{\psi, \phi}(0)\right\|
$$

for all $\phi, \psi$ as above. Our proof then consists of the following cases.

Case 1. If $D f(0)=0$ then $f$ is equivalent to (1)(iii) or (1)(iv).

Case 2. If $D f(0)$ can be chosen to have rank 1, then $f$ is equivalent to (1) (ii). Case 3. If $\|D f(0)(\alpha, \beta)\|=\rho\|(\alpha, \beta)\|$ for all $(\alpha, \beta) \in \mathbf{C}^{2}$ and some constant $\rho>0$ then $\rho=1$ and $f$ is equivalent to (1)(i). 
Case 4. Under the condition (11), if $D f(z, w)$ always has maximal rank then $\|D f(0)(\alpha, \beta)\| /\|(\alpha, \beta)\|$ is constant, $(\alpha, \beta) \neq(0,0)$.

The following lemma will be useful. Suppose $u \in \mathbf{C}^{n},\|u\|=1$, and let $H=\left\{Z \in \mathbf{C}^{n}:\langle Z, u\rangle=0\right\}$. Let $A \in B_{k}$ satisfy $\|A\|=1$.

Lemma 2. Suppose $f: B_{n} \rightarrow B_{k}$ is proper holomorphic and $f(\lambda u)=h(\lambda) A$, $|\lambda|<1$. Then $h(\lambda)$ is a finite Blaschke product and

$$
f(\lambda u+Z)=h(\lambda) A+p(\lambda, Z), \quad|\lambda|^{2}+\|Z\|^{2}<1, Z \in H,
$$

where $\langle p(\lambda, Z), A\rangle=0$.

Proof. The fact that $h$ is a finite Blaschke product follows from the fact that $\|f(\lambda u)\| \rightarrow 1$ as $|\lambda| \rightarrow 1$. Now set $p(\lambda, Z)=f(\lambda u+Z)-h(\lambda) A$ where $Z \in H$ and $|\lambda|^{2}+\|Z\|^{2}<1$. Now apply [2, Lemma 1] to conclude $\left\langle D^{l} f(\lambda u)(Z), A\right\rangle=0$ for $l=1,2, \ldots$. This completes the proof.

Now assume $n=2, k=3, P=L+Q+T, S=1+l+q$ where $L, Q$ and $T$ are homogeneous polynomials of degree 1,2 and 3 respectively and $l$ and $q$ are homogeneous of degree 1 and 2 respectively. Also, as remarked earlier, we may assume $L(z, w)=(a z, b w, 0)$ where $1 \geq a \geq b \geq 0$. With this notation, we have the following lemma.

Lemma 3. For all $(z, w) \in \mathbf{C}^{2}$,

$$
\langle P(z, w), T(\bar{w},-\bar{z})\rangle \equiv 0 .
$$

Further, if $a \geq b>0$, we may assume

$$
T(z, w)=\left(\frac{z}{a} q(z, w), \frac{w}{b} q(z, w), T_{3}(z, w)\right)
$$

where $T_{3}$ has one of the following forms

$$
\begin{aligned}
& \text { (i) } T_{3}(z, w)=r z q(z, w) \text { or } \\
& \text { (ii) } T_{3}(z, w)=r w l_{1} l_{2}^{*} \text { or } \\
& \text { (iii) } T_{3}(z, w)=r z l_{1}^{*} l_{2}^{*}=r z q^{*}
\end{aligned}
$$

where $r=\sqrt{1 / b^{2}-1 / a^{2}}$ and $q=l_{1} l_{2}$ with $l_{1}$ and $l_{2}$ linear.

Proof. If $u=(z, w),\|u\|=1$, then (12) is the same as (7). However, (12) is a holomorphic function for all $(z, w)$ and hence $(12)$ is true for all $(z, w) \in \mathbf{C}^{2}$. Equating coefficients of $\lambda^{2}$ in (4) yields $a \bar{z} T_{1}(z, w)+b \bar{w} T_{2}(z, w)=q(z, w)$ when $\|(z, w)\|=1$. For $z \neq 0$, replace $\bar{z}$ by $(1-w \bar{w}) / z$ to obtain

$$
\frac{a}{z} T_{1}(z, w)+\bar{w}\left(b T_{2}(z, w)-a \frac{w}{z} T_{1}(z, w)\right)=q(z, w), \quad\|(z, w)\|=1 .
$$

Since both sides of (15) are holomorphic in $z$ when $|z|>0$ it follows that equality holds for all $(z, w) \in \mathbf{C}^{2}, z \neq 0$. This implies $T_{1}=z q / a$ and 
$T_{2}=a w T_{1} / b z=w q / b$. The highest degree terms in (7) are $\left\langle T, \bar{T}^{*}\right\rangle \equiv 0$. Thus,

so

$$
\frac{1}{a^{2}} z w q q^{*}-\frac{1}{b^{2}} z w q q^{*}+T_{3}(z, w) T_{3}^{*}(z, w)=0
$$

$$
T_{3}(z, w) \overline{T_{3}(\bar{w},-\bar{z})}=\left(\frac{1}{b^{2}}-\frac{1}{a^{2}}\right) z w q(z, w) q^{*}(z, w)=r^{2} z w l_{1} l_{2} l_{1}^{*} l_{2}^{*} .
$$

Hence, either $l_{1}$ and $l_{2}$ are factors of $T_{3}$ or $l_{1}$ and $l_{2}^{*}$ are factors of $T_{3}$ or $l_{1}^{*}$ and $l_{2}^{*}$ are factors of $T_{3}$. Further, either $z$ or $w$ is a factor of $T_{3}$. Checking all the possibilities, we see that (14)(i), (ii) and (iii) are the only choices of $T_{3}$ (except for interchanging $l_{1}$ and $l_{2}$ ) that yield the correct value for $T_{3} T_{3}^{*}$.

Proof of the Main Theorem. Now assume $D f(0) \equiv 0$ so that $f=(Q+T) / S$. Since the left side of (4) has no $\lambda^{2}$ terms, it follows that $q \equiv 0$ so $s=1+l$. Now assume $T \not \equiv 0$. By replacing $f$ by $f \circ U$ where $U$ is unitary, if necessary, we may assume

$$
f(z, w)=\frac{1}{1+l} \sum_{k=2}^{3} \sum_{j=0}^{k} z^{k-j} w^{j} A_{k-j, j},
$$

$A_{30} \neq 0$ and $A_{03} \neq 0$. The equality $\langle P(u), P(u)\rangle=|1+l|^{2},\|u\|=1$ implies that $\left\langle A_{30}, A_{03}\right\rangle=0$ (i.e. the $z_{3} \bar{w}^{3}$ term must drop out). Set $E_{1}=A_{30} /\left\|A_{30}\right\|$ and $E_{3}=A_{03} /\left\|A_{03}\right\|$ and let $E_{2}$ be a unit vector in $\mathrm{C}^{3}$ that is orthogonal to both $E_{1}$ and $E_{3}$ so that $\left\{E_{1}, E_{2}, E_{3}\right\}$ is an orthonormal basis for $\mathbf{C}^{3}$. We now use

$$
\langle P(z, w), P(z, w)\rangle=|1+l(z, w)|^{2}, \quad\|(z, w)\|=1,
$$

and

$$
\left\langle Q(z, w), \overline{T^{*}(z, w)}\right\rangle=0 .
$$

From (16), we see that $\left\langle A_{30}, A_{02}\right\rangle=0$ and $\left\langle A_{03}, A_{20}\right\rangle=0$. From (17), $\left\langle A_{30}, A_{20}\right\rangle=0$ and $\left\langle A_{03}, A_{02}\right\rangle=0$. Now using (16) again, we get $\left\langle A_{30}, A_{11}\right\rangle=$ 0 and $\left\langle A_{03}, A_{11}\right\rangle=0$. Thus, $Q(z, w)=h(z, w) E_{2}$ where $h$ is a scalar homogeneous polynomial of degree 2 . By use of a unitary map in $\mathbf{C}^{2}$ we may assume $A_{02}=0$. This may necessitate redefining $A_{03}$ and $A_{30}$ but $E_{2}$ will remain as before with $\left\langle A_{30}, E_{2}\right\rangle=0=\left\langle E_{2}, A_{03}\right\rangle$. Now setting $z=0$, we see that (aside from a constant scalar of modulus 1$), f(0, w)=w^{3} E_{3}$. Using Lemma 2, we conclude $l(z, w) \equiv 0$. By proper choice of coordinates we now may assume $f(z, w)=\left(T_{1}, T_{2}+Q_{2}, w^{3}\right)$ where $z$ is a factor of $T_{1}, T_{2}$ and $Q_{2}$ and $w$ is a factor of $T_{2}$. Using (17), we see that either $T_{2} \equiv 0$ or $Q_{2} \equiv 0$. Now $Q_{2} \equiv 0$ implies $T_{1}=z^{3}$ (to see this, set $w=0$ ) but then $|z|^{6}+\left|T_{2}\right|^{2}+|w|^{6}$ cannot be 1 on $\partial B_{2}$ so we must have $T_{2} \equiv 0$. Now $Q_{2}=z(\alpha z+\beta w)$ for some $\alpha$ and $\beta$. Using (16), we see that $T_{1}=c z^{k} w^{3-k}$ for some $c$ and $k, 1 \leq k \leq 3$, and 
either $\alpha=0$ or $\beta=0$. It is routine to check that $k=3,|c|=1, \alpha=0$, $|\beta|=\sqrt{3}$ is the only possiblity. That is $f$ is equivalent to (1)(iv).

Now assume $D f(0)=0$ and $T \equiv 0$. From (4), it is easy to see that $S \equiv 1$ and $f=Q$. In the same notation as above, it is trivial to see that $\left\langle A_{20}, A_{02}\right\rangle=0=\left\langle A_{20}, A_{11}\right\rangle=\left\langle A_{11}, A_{02}\right\rangle$. Further, it then readily follows that $f$ is equivalent to (1)(iii).

We have completed Case 1 of the previous section.

We now proceed to Case 2 assuming $D f(0)$ has rank 1. By proper choice of coordinates, $L(z)=(a z, 0,0), 1 \geq a>0$. Since $T_{1} a \bar{z}=q$ (i.e. from (4), the coefficients of $\lambda^{2}$ must be equal), $|z|^{2}+|w|^{2}=1$ and both sides are holomorphic in $w$, the equality persists for all $(z, w)$. Hence $T_{1} \equiv 0 \equiv q$. Thus,

$$
f=\frac{1}{1+l}\left(a z+Q_{1}, Q_{2}+T_{2}, Q_{3}+T_{3}\right) .
$$

Write

$$
P(z)=a z A_{10}+\sum_{k=2}^{3} \sum_{j=0}^{k} z^{k-j} w^{j} A_{k-j, j} .
$$

If $T \equiv 0$, then using (4) it is easy to see that $Q_{1} \equiv 0 \equiv l$. Setting $z=0$, it easily follows that $\left\|A_{02}\right\|=1$ so by proper choice of coordinates using Lemma 2, we may assume $Q_{3}=w^{2}$. It is then straightforward to conclude that $Q_{2}=$ $c z^{k} w^{2-k}, k=1$ or $k=2$. Then it follows readily that $k=1, a=1$ and $|c|=1$ so $f$ is equivalent to (1)(ii).

Now assume $T \not \equiv 0$. As before, $\left\langle A_{30}, A_{03}\right\rangle=0$ and after possibly composing with a unitary map, we may assume $z$ is a factor of $T_{2}$ and $w$ is a factor of $T_{3}$. Set $T_{2}=z l_{1} l_{2}$ where $l_{1}$ and $l_{2}$ are linear. Using (7), we conclude $T_{2} T_{2}^{*}+T_{3} T_{3}^{*}=0$. It then follows that either $T_{3}=w l_{1} l_{2}$ or $T_{3}=w l_{1}^{*} l_{2}^{*}$. Now fix $u=(z, w),\|u\|=1$. Using [2, Theorem 2], there exist constants $c_{1}, c_{2}, c_{3}$ (not all 0) such that $c_{1} P_{1}(\lambda u)+c_{2} P_{2}(\lambda u)+c_{3} P_{3}(\lambda u)=0,|\lambda|<1$. Here we may assume $c_{1}, c_{2}$ and $c_{3}$ vary continuously with $u=(z, w)$. Divide by $\lambda$ and let $\lambda \rightarrow 0$ to see that $c_{1}=0$. Then $c_{2} T_{2}(u)+c_{3} T_{3}(u)=0$ and $c_{2} Q_{2}(u)+c_{3} Q_{3}(u)=0$.

Assuming $T_{3}=w l_{1} l_{2}$, we may take $c_{3}=-z, c_{2}=w$. Then it follows that $w$ is a factor of $Q_{3}$ and $z$ is a factor of $Q_{2}$. In fact, $P_{2} / z=P_{3} / w$. Then the map

$$
h(z, w)=\frac{1}{1+l}\left(a z+Q_{1}, \frac{P_{2}}{z}\right)
$$

is a proper mapping with $h(0,0)=(0,0)$. This implies that $h$ is unitary so $l \equiv 0, Q_{1} \equiv 0, a=1 . P_{2} / z=w$ (except for a possible rotation). In this case, $f$ is equivalent to $(1)(\mathrm{ii})$.

Now assume $T_{3}=w l_{1}^{*} l_{2}^{*}$. We may take $c_{2}=-Q_{3}(u), c_{3}=Q_{2}(u)$ so that $Q_{3} z l_{1} l_{2}=Q_{2} w l_{1}^{*} l_{2}^{*}$. It is readily seen that $l_{1}^{*}$ is not a multiple of $l_{1}$. If $l_{1}^{*}$ is a multiple of $l_{2}$ then $l_{2}^{*}$ is a multiple of $l_{1}$ and we actually have $T_{3}=w l_{1} l_{2}$ again. If neither $l_{1}^{*}$ nor $l_{2}^{*}$ is a multiple of $z$, then $w l_{1}^{*} l_{2}^{*}$ is a factor of $Q_{3}$ and this 
is impossible since $\operatorname{deg} Q_{3}=2$. Thus, we may assume $l_{2}=w$ and $l_{2}^{*}=-z$. Then $l_{1}$ is a factor of $Q_{2}$ and $l_{1}^{*}$ is a factor of $Q_{3}$. It then follows that $Q_{2}$ and $Q_{3}$ have a common factor $l_{3}$ and $P_{2}=l_{1}\left(l_{3}+z w\right)$ and $P_{3}=l_{1}^{*}\left(l_{3}+z w\right)$. If $l_{1}=\alpha z+\beta w$, then $\left|l_{1}\right|^{2}+\left|l_{1}^{*}\right|^{2}=|\alpha|^{2}+|\beta|^{2}$ when $|z|^{2}+|w|^{2}=1$ and it follows that

$$
h(z, w)=\frac{1}{1+l}\left(P_{1}, \frac{P_{2}}{\sqrt{|\alpha|^{2}+|\beta|^{2} l_{1}}}\right)
$$

is proper with $h(0,0)=0$. This again means $h$ is unitary so (up to a rotation $\left.w \rightarrow w e^{i \phi}\right), h(z, w)=(z, w)$. Then,

$$
a=1, \quad Q_{1}=z l, \quad \frac{Q_{2}+T_{2}}{1+l}=w z \quad \text { and } \quad \frac{Q_{3}+T_{3}}{1+l}=w^{2} .
$$

Hence $f$ is equivalent to (1)(ii). This completes Case 2.

Now assume $\|D f(0)(\alpha, \beta)\|=\rho\|(\alpha, \beta)\|$ for all $(\alpha, \beta) \in \mathbf{C}^{2}$ where $0<\rho \leq$ 1. In this case, we may take $L(z, w)=(a z, a w, 0), a=\rho$. By Lemma 3, $T_{3}=0$ and $T_{1}=z q / a, T_{2}=w q / a$. Using (7), we see that $\left\langle Q, \bar{T}^{*}\right\rangle \equiv 0$ so $Q_{1} w q^{*} / a-Q_{2} z q^{*} / a \equiv 0$. Therefore $Q_{2}=w Q_{1} / z$.

We now see that $f_{2}=w f_{1} / z$ and it follows that $(z, w) \rightarrow\left(f_{1} / z, f_{3}\right)$ is a proper map of $B_{2}$ into $B_{2}$ hence is of degree 1 (or is constant). However the degree of this map is at least as large as $\operatorname{deg} Q_{3} / S=2$ unless $Q_{3} \equiv 0$. Therefore, $Q_{3} \equiv 0, a=1$ and $f$ is equivalent to $(1)(\mathrm{i})$.

One case remains. We now assume $D f(0)$ has rank 2 ,

$$
\|D f(0)(\alpha, \beta)\| /\|(\alpha, \beta)\|
$$

is not constant, $(\alpha, \beta) \neq(0,0)$, and that $\|D f(0)\| \leq\left\|D g_{\psi \phi}(0)\right\|$ for all $\psi, \phi$ holomorphic automorphisms of $B_{3}$ and $B_{2}$ respectively. Let $\phi$ and $\psi$ be given by (9). Then,

$$
D \phi(0)=I+O\left(r^{2}\right) \text { and } D \psi(f(r u))=I+O\left(r^{2}\right)
$$

where $I$ is the identity on the appropriate space. Therefore,

$$
D \psi \circ f \circ \phi(0)=D f(r u)+o(r) .
$$

We may assume $L(z, w)=(a z, b w, 0)$ where $0<b<a \leq 1$. We wish to show that the minimal norm property implies that the quadratic part $Q$ of $f$, has the form $Q_{1}(u)=a u_{1} l(u)+a_{02} u_{2}^{2}$ for some $a_{02}$. To that end, we first show that we need only consider $(z, w),|z|^{2}+|w|^{2}=1$ with $|w|<\varepsilon$ where $\varepsilon$ is small and positive. Set $L_{r u}=D f(r u)$. We know $a=\|L\| \leq\left\|L_{r u}\right\|$. We have $L_{r u}(z, w)=(a z+O(r), b w+O(r), O(r))$. Let $\varepsilon>0$ and $|w| \geq \varepsilon$. Clearly, there exists $r(\varepsilon)>0$ and $\delta>0$ such that $r<r(\varepsilon)$ implies

$$
\begin{aligned}
\left\|L_{r u}(z, w)\right\|^{2} & =a^{2}|z|^{2}+b^{2}|w|^{2}+O(r)<a^{2}-\delta \\
& <\|L\|^{2} \text { when }|z|^{2}+|w|^{2}=1, \quad|w| \geq \varepsilon .
\end{aligned}
$$


Hence we assume $|w|<\varepsilon, \varepsilon$ to be chosen later. Then

$$
\begin{aligned}
\left\|L_{r u}(z, w)\right\|^{2}=\left|z \frac{\partial f_{1}}{\partial z}(r u)\right|^{2}+O_{1}(\varepsilon)+O_{2}\left(r^{2}\right) \\
=\left|\left[(1+r l(u))\left(a z+r \frac{\partial Q_{1}}{\partial z}(u) z\right)-a r u_{1} l(z, 0)\right](1-2 r l(u))\right|^{2} \\
\quad+O_{1}(\varepsilon)+O_{2}\left(r^{2}\right) \\
=|z|^{2}\left(a^{2}+2 a r \operatorname{Re}\left[\frac{\partial Q_{1}}{\partial z}(u)-2 a l(u)+a l\left(0, u_{2}\right)\right]\right. \\
\quad+O_{1}(\varepsilon)+O_{2}\left(r^{2}\right)<a^{2}
\end{aligned}
$$

for sufficiently small $\varepsilon$ and $r$ for appropriate choice of $u$ unless $\partial Q_{1}(u) / \partial z=$ $2 a l(u)-a l\left(0, u_{2}\right)$. That is,

$$
Q_{1}(u)=a u_{1} l(u)+a_{02} u_{2}^{2}
$$

for some $a_{02}$.

Now assume $q=l_{1} l_{2}$ and consider the fifth degree terms of (7). By Lemma 3 , there are three possibilities for $T_{3}$. Assume first $T_{3}=r z l_{1} l_{2}$ and $r=$ $\sqrt{1 / b^{2}-1 / a^{2}}$. We have

$$
Q_{1} \frac{w}{a} l_{1}^{*} l_{2}^{*}-Q_{2} \frac{z}{b} l_{1}^{*} l_{2}^{*}+Q_{3} r l_{1}^{*} l_{2}^{*}=0
$$

Therefore, $w$ is a factor of $Q_{2}$ so $Q_{2}=w l_{3}$. Then $Q_{1}=a z l_{3} / b-a r Q_{3}$.

If we replace $f$ by $U \circ f$ where $U$ is the unitary map

then $f$ has the form

$$
\left(\begin{array}{ccc}
\frac{b}{a} & 0 & b r \\
0 & 1 & 0 \\
-b r & 0 & \frac{b}{a}
\end{array}\right)
$$

$$
\frac{1}{S}\left(b z+z l_{3}+\frac{z}{b} q, b w+w l_{3}+\frac{w}{b} q,-a b r z-a r z l_{3}+\left(\frac{b}{a}+a b r^{2}\right) Q_{3}\right) .
$$

Since $f_{1} / z=f_{2} / w$, the map $(z, w) \rightarrow\left(f_{1} / z, f_{3}\right)$ is proper and therefore of degree 1 . This means $S, P_{1}, P_{2}$ and $P_{3}$ have a common factor so we may take $S=1+l$ and $f=\left(b z+z l_{4}, b w+w l_{4},-a b r z\right) /(1+l)$. Using (4) and equating the coefficients of $\lambda$ we get $l_{4}=l / b$.

If $b=1$, then $a=1$ and $f=(z, w, 0)$. If $b<1$, setting $z=0$ we see that $\left|\left(b+\frac{1}{b} l(0, w)\right) /(1+l(0, w))\right|=1$ when $|w|=1$. Hence by Lemma 2, $l(z, w)=l(0, w)$ and in fact after a possible rotation, $l(z, w)=b w$. Thus,

$$
f=\left(z \frac{b+w}{1+b w}, w \frac{b+w}{1+b w}, \frac{-\sqrt{a^{2}-b^{2}} z}{1+b w}\right) .
$$

Setting $w=0$, we see that $a=1$ and replacing $f$ by $V \circ f$ where

$$
V=\left(\begin{array}{ccc}
b & 0 & -\sqrt{1-b^{2}} \\
0 & 1 & 0 \\
\sqrt{1-b^{2}} & 0 & b
\end{array}\right)
$$


we get

$$
f=\left(z, \frac{w(b+w)}{1+b w}, \frac{\sqrt{1-b^{2}} z w}{1+b w}\right) .
$$

It is easy to see that $\operatorname{Df}(0, w)$ has rank 1 for an appropriate choice of $w$ and this case has already been considered.

Now consider $T_{3}=r w l_{1} l_{2}^{*}$.

Using (7) as before, we get

$$
Q_{1} \frac{w}{a} l_{1}^{*} l_{2}^{*}-Q_{2} \frac{z}{b} l_{1}^{*} l_{2}^{*}+Q_{3} r z l_{1}^{*} l_{2}=0
$$

Therefore, we may assume $Q_{1}=a z l_{3}$ (if $z$ is a factor of $l_{2}^{*}$ then $w$ is a factor of $l_{2}$ and this case would be identical to the previous Case 1). Thus, $w l_{3} l_{2}^{*}-Q_{2} l_{2}^{*} / b+r Q_{3} l_{2}=0$ so $Q_{3}=l_{2}^{*} l_{4}$. It follows that $Q_{2}=b r l_{2} l_{4}+b w l_{3}$. Due to our assumption on the minimality of $\|L\|=\|D f(0)\|$, we have $a z l_{3}=$ $a z l+a_{02} w^{2}$ so $a_{02}=0$ and $l_{3}=l$.

Equating coefficients of $\lambda$ in (4) yields

$$
\begin{aligned}
& \frac{z}{a} q a \overline{z l}+\frac{w}{b} q\left(b \overline{w l}+b r \overline{l_{2} l_{4}}\right)+r w l_{1} l_{2}^{*}\left(\overline{l_{2}^{*} l_{4}}\right) \\
& \quad+a|z|^{2} l+b \bar{w}\left(b w l+b r l_{2} l_{3}\right)=q \bar{l}+l \quad \text { when }|z|^{2}+|w|^{2}=1 .
\end{aligned}
$$

The first and second terms on the left clearly cancel with the first term on the right. Now setting $w=0,|z|=1$ yields $a^{2} l(z, 0)=l(z, 0)$ so that either $a=1$ or $l(z, w)=d w$ for some $d$. In case $a=1, f_{1}(z, w) \equiv z$ by Lemma 2 and $w$ is a factor of $f_{2}$ and $f_{3}$. Then, $(z, w) \rightarrow\left(f_{2} / w, f_{3} / w\right)$ is a proper map of $B_{2}$ onto $B_{2}$ hence of degree 1 . Then we may assume

$$
f(z, w)=\left(z, \frac{w(b+\alpha z+\beta w)}{1+b(\alpha z+\beta w)}, \frac{w \sqrt{1-b^{2}}(\bar{\alpha} w-\bar{\beta} z)}{1+b(\alpha z+\beta w)}\right)
$$

where $|\alpha|^{2}+|\beta|^{2}=1$ (up to composition by unitary maps in $B_{2}$ and $B_{3}$ ). One can then check to see that $D f\left(-\bar{\alpha} b, b \bar{\beta} \sqrt{1-b^{2}|\alpha|^{2}} /\left(\sqrt{1-b^{2}|\alpha|^{2}}+\sqrt{1-b^{2}}\right)\right.$ has rank one contradicting our assumption.

Thus, we assume $l(z, w)=d w$ and (19) becomes

$$
q r \overline{l_{2} l_{4}}+r l_{1}\left|l_{2}^{*}\right|^{2} \bar{l}_{4}+a|z|^{2} d+b^{2}|w|^{2} d+b^{2} \frac{\bar{w}}{w} r l_{2} l_{4}=d \text {. }
$$

As previously remarked, $w$ does not divide $l_{2}$ (this would reduce to a previous case) so $w$ divides $l_{4}$.

Setting $w=0$ then yields $a=1$ or $d=0$. Since $a=1$ was completed above, we assume $d=0$ so $l=0$ and $S=1+l_{1} l_{2}$. Since we now know $w$ is a factor of $f_{2}$ and of $f_{3}, f_{1}(z, w)=f_{1}(z, 0)$ by Lemma 2. Hence $a=1$ (already considered) or $l_{1} l_{2}=a z^{2}$. Thus, we assume

$$
f(z, w)=\frac{1}{1+a z^{2}}\left(z\left(a+z^{2}\right), w\left(b+c z+\frac{a}{b} z^{2}\right), r a w^{2} z+\frac{c}{b r} w^{2}\right) .
$$


Again using $\langle T, Q\rangle+\langle Q, L\rangle=q \bar{l}+l,|z|^{2}+|w|^{2}=1$, we get $a \bar{c} / b+c b=0$. This is only possible when $c=0$ since $0<b<a<1$.

On $\partial B_{2}$, the sixth degree terms of $|P|^{2}-|S|^{2}$ are now

$$
|z|^{6}-|z|^{2}|w|^{4}+\frac{a^{2}}{b^{2}}|w|^{2}|z|^{2}\left(|z|^{2}+|w|^{2}\right)=|z|^{4}-|z|^{2}|w|^{2}+\frac{a^{2}}{b^{2}}|z|^{2}|w|^{2} .
$$

Then the fourth degree terms are $\left(1-a^{2}\right)|z|^{4}+\left(a^{2} / b^{2}-1\right)|z|^{2}|w|^{2}$, and thus it must be true that $1-a^{2}=a^{2} / b^{2}-1$. This is $b^{2}=a^{2} /\left(2-a^{2}\right)$. The second degree terms now become

$$
\left(1-a^{2}\right)|z|^{2}+a^{2}|z|^{2}+b^{2}|w|^{2}=|z|^{2}+b^{2}|w|^{2} .
$$

Therefore $1=b=a$ and $f$ is equivalent to (1)(i).

Finally, we consider case (iii). Set $q(z, w)=\alpha z^{2}+\beta z w+\gamma w^{2}=l_{1} l_{2}$ so that $l_{1}^{*} l_{2}^{*}=\bar{\alpha} w^{2}-\bar{\beta} z w+\bar{\gamma} z^{2}$. Note that $l_{1}^{*}(z, w)=0$ and $l_{1}(z, w)=0$ for the same $(z, w)$ implies $(z, w)=0$ unless $l_{1} \equiv 0$. Further, if $l_{1}^{*}$ and $l_{2}$ have the same zero set, then so do $l_{2}^{*}$ and $l_{1}$, so that $l_{1} l_{2}=l_{1}^{*} l_{2}^{*}$ (up to multiplication by a constant) and this would again be case (i) which is complete. Also, if $z$ divides $l_{1}$ then $w$ divides $l_{1}^{*}$ so $z l_{1}^{*} l_{2}^{*}=w l_{1} l_{2}^{*}$ (up to multiplication by a constant) and this is case (ii). Thus, we may assume

$$
q(z, w)=\alpha z^{2}+\beta z w+\gamma w^{2} \quad \text { and } \quad q^{*}(z, w)=\bar{\alpha} w^{2}-\bar{\beta} z w+\bar{\gamma} z^{2}
$$

have no common factors and $\gamma \neq 0$. Proceeding as before, using the fifth degree terms of (7),

$$
Q_{1} \frac{w q^{*}}{a}-Q_{2} \frac{z q^{*}}{b}+Q_{3} r w q=0
$$

It clearly follows that $w$ is a factor of $Q_{2}$ and that $Q_{3}=c q^{*}$ for some $c$. Set $Q_{2}=b w l_{3}$. Then $Q_{1}=a z l_{3}-a c r q=a z l+\delta w^{2}$ using the minimal property of $\|D f(0)\|$. Then $\delta=-a c r \gamma$ and

$$
l_{3}=l+c r\left(\frac{q-\gamma w^{2}}{z}\right)=l+c r(\alpha z+\beta w) .
$$

We will take $l=d z+e w$.

Proceeding as before $\langle T, Q\rangle+\langle Q, L\rangle=q \bar{l}+l$ on $\partial B_{2}$ so that

$$
\begin{aligned}
|z|^{2} q \bar{l} & +\frac{z}{a} q \bar{\delta} \bar{w}^{2}+|w|^{2} q \bar{l}+|w|^{2} q \bar{c} r(\overline{\alpha z+\beta w}) \\
& +r z \bar{c}\left|q^{*}\right|^{2}+a^{2}|z|^{2} l+a \delta w^{2} \bar{z}+b^{2}|w|^{2} l \\
& +b^{2} c r|w|^{2}(\alpha z+\beta w)=q \bar{l}+l \quad \text { when }|z|^{2}+|w|^{2}=1
\end{aligned}
$$


The first and third terms on the left cancel with the first term on the right so (replacing $\bar{z}$ by $(1-w \bar{w}) / z)$ we get

$$
\begin{aligned}
\frac{z}{a} q \bar{\delta} & \bar{w}^{2}+|w|^{2} q \bar{c} r\left(\bar{\alpha} \frac{1-w \bar{w}}{z}+\bar{\beta} \bar{w}\right) \\
& +r \bar{c} z\left(\bar{\alpha} w^{2}-\bar{\beta} z w+\bar{\gamma} z^{2}\right)\left(\alpha \bar{w}^{2}-\beta \bar{w} \frac{1-w \bar{w}}{z}+\gamma \frac{1-2|w|^{2}+|w|^{4}}{z^{2}}\right) \\
& +\left(b^{2}-a^{2}\right)|w|^{2} l+a \delta w^{2} \frac{1-w \bar{w}}{z} \\
& +b^{2} c r|w|^{2}(\alpha z+\beta w)=\left(1-a^{2}\right) l .
\end{aligned}
$$

Since both sides are holomorphic in $z, z \neq 0$, they must be equal for all $z \neq 0$. The terms that have $\bar{w}$ as a factor (but not $w$ or $\bar{w}^{2}$ ) are $-r \bar{\gamma} \beta \bar{c} z^{2}=0$. Since $\gamma \neq 0$ and $r \neq 0$, either $\beta=0$ or $c=0$. However, $c=0$ implies $\delta=0$ and $\left(b^{2}-a^{2}\right)|w|^{2}=1-a^{2}$ so $a=1=b$. Hence we must have $\beta=0$. The $w$ terms are $-\bar{\beta} r \gamma=\left(1-a^{2}\right) e$. Since $a \neq 1, \beta=0$, we get $e=0$. The coefficient of $1 / z$ is now

$$
\bar{\alpha} \bar{c} r \gamma\left(|w|^{2}-|w|^{4}\right) w^{2}+r \bar{c} \bar{\alpha} \gamma\left(1-2|w|^{2}+|w|^{4}\right) w^{2}-a^{2} \operatorname{cr} \gamma\left(1-|w|^{2}\right) w^{2}=0 .
$$

Thus, it follows that $r \bar{c} \bar{\alpha} \gamma=a^{2} c r^{\prime} \gamma$ from which $\alpha=\bar{c} a^{2} / c=a^{2}$ (because we may assume $c>0$ by rotation).

The coefficient of $\bar{w}^{2}$ is $\alpha z^{3} / a+r \alpha \bar{c} \bar{\gamma} z^{3}=0$ so that $r c \gamma=-1 / a$. That is $\gamma=-1 / \operatorname{arc}$ and we observe that $\delta=1$. Now setting $z=0,|w|=1$, we observe that

$$
\|f(0, w)\|^{2}=\frac{1}{|1+\gamma w|^{2}}\left[|w|^{2}+\left|b w+\frac{\gamma w^{3}}{b}\right|^{2}+\left|c \bar{\alpha} w^{2}\right|^{2}\right]=1
$$

so that

$$
1+b^{2}+2 \operatorname{Re} \gamma w^{2}+\frac{|\gamma|^{2}}{b^{2}}+|c|^{2}|\alpha|^{2}=1+2 \operatorname{Re} \gamma w^{2}+|\gamma|^{2} .
$$

Hence $b^{2}+|\gamma|^{2} / b^{2}+|c|^{2}|\alpha|^{2}=|\gamma|^{2}$. This is not possible because $|\gamma|^{2} / b^{2}>|\gamma|^{2}$.

Our proof is now complete.

\section{REFERENCES}

1. H. Alexander, Proper holomorphic mappings in $\mathbf{C}^{n}$, Indiana Univ. Math. J. 26 (1977), 137146.

2. J. Cima and T. Suffridge, A reflection principle with applications to proper holomorphic mappings, Math. Ann. 265 (1983), 489-500.

3. J. Faran, Maps from the two-ball to the three-ball, Invent. Math. 68 (1982), 441-475.

4. _ The linearity of proper holomorphic maps between balls in the low codimension case, J. Differential Geometry 24 (1986), 15-17.

5. S. Webster, On mapping an $n$-ball into an $n+1$-ball in complex space, Pacific J. Math. 81 (1979), 267-272.

Department of Mathematics, University of North Carolina, Chapel Hill, North CarOLINA 27514

Department of Mathematics, University of Kentucky, LeXington, Kentucky 40506 